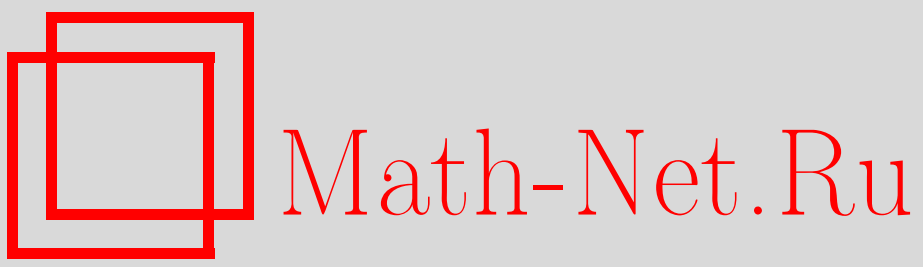

Zhong Gen Su, On the weak convergence of vector-valued continuous random processes, Теория вероятн. и ее примен., 1998, том 43, выпуск 3, 561-576

DOI: https://doi.org/10.4213/tvp1560

Использование Общероссийского математического портала Math-Net.Ru подразумевает, что вы прочитали и согласны с пользовательским соглашением http://www.mathnet.ru/rus/agreement

Параметры загрузки:

IP : 107.22 .136 .117

26 апреля 2023 г., 03:22:37

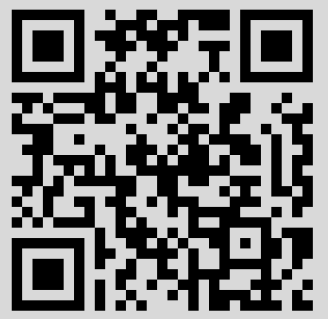




\title{
ON THE WEAK CONVERGENCE OF VECTOR-VALUED CONTINUOUS RANDOM PROCESSES ${ }^{1)}$
}

\begin{abstract}
В статье устанавливаются некоторые результаты о слабой сходимости непрерывных векторнозначных случайных процессов, таких, например, как векторнозначные субгауссовские процессы, векторнозначные процессы, удовлетворяюшие условию Липшица, и случайные ряды Фурье с векторнозначными коэффициентами. Полученные результаты могут рассматриваться как обобшение соответствуюших результатов на вещественной прямой. Наши основные идеи берут началіо в [3] и [4].
\end{abstract}

Ключевые слова и фразы: слабая сходимость, векторнозначные гауссовские процессы, метрическая энтропия, пространство типа 2.

1. Introduction and preliminary statements. Suppose that $(T, d)$ is a compact metric or pseudometric space, $(B,\|\cdot\|)$ is a separable real or complex Banach space with its topological dual $B,\langle\cdot, \cdot\rangle$ denoting their natural bilinear form. Let $C(T, B)$ be the linear space of all continuous functions on $(T, d)$ with values in $B$. In particular, $C(T)$ represents either $C(T, \mathbf{R})$ or $C(T, \mathbf{C})$. Then $C(T, B)$ is a separable Banach space with the natural sup-norm $\|x\|=\sup _{t \in T}\|x(t)\|$. Our aim of this article is to discuss the weak convergence of a sequence of random processes with sample paths in $C(T, B)$. Fernique [4] proved the following characterization of relatively compact subsets in $C(T, B)$.

Proposition 1.1. Suppose that $F$ is a separating set of real continuous functions on $B$. For a subset $C$ of $C(T, B)$ to be relatively compact it is sufficient and necessary that the set $C$ satisfies

(i) there is a compact set $K$ of $B$ such that $\{x(t), t \in T, x \in C\} \subset K$,

(ii) for each $f \in F$, the set $f \circ C=\{f \circ x, x \in C\}$ is relatively compact in $C(T)$.

We endow $C(T, B)$ with a Borel $\sigma$-field $\mathscr{B}(C(T, B))$ induced by the uniform topology, then the well-known Prokhorov theorem holds for any family of measures on $(C(T, B), \mathscr{B}(C(T, B)))$ and from Proposition 1.1 we easily obtain

*Department of Mathematics, Hangzhou University, 310028, China; Research Institute of Mathematics, Fudan University, 200034 Shanghai, China. Province.

1) This project is supported by Natural Science Foundations of China and Zhejiang 
Proposition 1.2. Let $\mathscr{M}$ be a family of probability measures on the space $C(T, B)$. Suppose that for any positive $\varepsilon$ there exists a compact set $K_{\varepsilon}$ of $B$ such that

$$
\mu\left\{x: \exists t \in T, x(t) \notin K_{\varepsilon}\right\} \leqslant \varepsilon, \quad \forall \mu \in \mathscr{M} .
$$

Then $\mathscr{M}$ is relatively compact if and only if there exists a separating set $F$ of real continuous functions on $B$ such that for any $f \in F$ the family

$$
f \circ \mathscr{M}=\{f \circ \mu, \mu \in \mathscr{M}\}
$$

is relatively compact on $C(T)$. Here $f \circ \mu(A)=\mu\{x \in C(T, B), f \circ x \in A\}$ for each Borel subset $A$ in $C(T)$.

Since it is easy for us to deal with the relative compactness of a family of probability measures on $C(T)$, the key to the relative compactness of a family of probability measures on $C(T, B)$ is to verify the first condition of Proposition 1.2. We will base our discussions on Proposition 1.2 in two ways. One way is to use the following facts: a subset $K$ of a separable Banach space $B$ is relatively compact if and only if $K$ is bounded and for any $\eta>0$ there is a finite-dimensional closed subspace $F$ such that $q_{F}(x) \leqslant \eta$ for all $x \in K$, where $q_{F}$ denotes the quotient norm, i.e., $q_{F}(x)=\inf _{y \in F}\|x-y\|$. Thus, we can rewrite (1.1) of Proposition 1.2 as follows.

For any $\varepsilon>0$ and $\eta>0$ there are a positive constant $M$ and a finitedimensional closed subspace $F$ such that

$$
\mu\{x: \exists t \in T,\|x(t)\|>M\}<\varepsilon
$$

and

$$
\mu\left\{x: \exists t \in T, q_{F}(x(t))>\eta\right\}<\varepsilon
$$

for any $\mu \in \mathscr{M}$.

The second way needs something more. Let $C$ be a convex symmetric closed set in $B$ with its gauge $N_{C}(x)=\inf \{\lambda: x \in \lambda C\}$ and polar set $C^{0}=\left\{f \in B^{\prime}:\langle f, x\rangle \leqslant 1\right.$, for all $\left.x \in C\right\}$. If $C$ is convex and $\sigma\left(B, B^{\prime}\right)$ closed, we have $C=\cap_{y \in C^{\circ}} A(y)$ where $A(y)=\{x:\langle y, x\rangle \leqslant 1\}$. Clearly, $C^{c}=\cup_{y \in C^{\circ}} A(y)^{c}$. Thus there exists a sequence of $y_{n}$ such that for any $x \in B$,

$$
N_{C}(x)=\inf \{\lambda>0: x \in \lambda C\}=\sup _{n}\left\langle y_{n}, x\right\rangle .
$$

On the other hand, suppose that $b$ is a closed and convex symmetric bounded subset of $B$, then $b$ is the intersection of a sequence of decreasing convex symmetric and closed neighborhood of origin in $B$, and hence there exists a sequence of increasing continuous seminorms $N_{k}$, converging simply to $N_{b}$, in $B . B$ with such a continuous seminorm $N_{k}$ defines a separable Banach space $B_{k}=\left(B, N_{k}\right)$ by means of quotient and completion. We are also to work with these Banach spaces as well as $(B,\|\cdot\|)$. 
Proposition 1.2 concerns only the relative compactness, the following Singer theorem can usually be used to identify the candidate for the limit in weak convergence of a family of probability measures.

Theorem (I. Singer, [11]). The topological dual of $C(T, B)$ is isometrically isomorphic to the space of all the completely additive and regular functions $f_{e}$ defined on the Borel set e $\subset T$ with values in $B^{\prime}$. The isomorphism $\Phi \leftrightarrow f_{e}$ between the two spaces is given by the relation

$$
\Phi(x)=\int\left\langle x(t), d f_{t}\right\rangle, \quad \forall x \in C(T, B) .
$$

As in the scalar setting, let $\mathscr{W}=\left\{\sum_{i=1}^{n} f_{i} \delta_{t_{i}}, f_{i} \in B^{\prime}, t_{i} \in T, 1 \leqslant i \leqslant n\right.$, $n \geqslant 1\}$, where $\delta_{t}$ denotes the unit point mass at $t$, then $\mathscr{W}$ is sequentially $w^{*}$-dense in the topological dual $(C(T, B))^{\prime}$ of $C(T, B)$.

Next let us introduce some notation. Throughout we assume that $(\Omega, \mathscr{F}, \mathbf{P})$ is a complete probability space on which all random variables are defined. A random process with values in $B$ is a family $X=(X(t), t \in T)$ of measurable mappings from $(\Omega, \mathscr{F}, \mathbf{P})$ to $(B, \mathscr{B})$ indexed by $T$. Two random processes $X$ and $Y$ are modifications each other if for each $t \in T$, $\mathbf{P}\{X(t)=Y(t)\}=1, X=(X(t), t \in T)$ is said to be Gaussian if the family $\left\{\langle X(t), y\rangle, t \in T, y \in B^{\prime}\right\}$ is a family of zero-mean real Gaussian random variables. Thus a $B$-valued Gaussian process $X$ is uniquely determined by its covariance structure $\mathrm{E}\langle X(t), y\rangle\langle X(s), z\rangle$. As an example we give the construction of $B$-valued Wiener process generated by a given probability measure $\mu$. Let $T=\left[0, T_{0}\right]$; for any $t \in T, R_{t}=R_{t}(y, z)$ is a symmetric semi-positive bilinear form on $B^{\prime}$. Assume that for each $y \in B^{\prime}$, the mapping $t \rightarrow R_{t}(y, y)$ is increasing and right-continuous. We also assume that there exists a Gaussian probability measure $\mu$ on $B$ such that

$$
\int\langle x, y\rangle^{2} d \mu(x)=R_{T_{0}}(y, y) \quad \forall y \in F
$$

Then, under these conditions, there really exists a Gaussian random process $W$ indexed by $T$ with values in $B$ such that for all $s, t \in T$ and $y, z \in B^{\prime}$,

$$
\mathbf{E}\langle W(s), y\rangle\langle W(t), z\rangle=R_{s \wedge t}(y, z) .
$$

This assertion can be proved as follows.

For any pair $(s, t)$ with $s<t$, the difference $R_{t}-R_{s}$ is the covariance of a Gaussian measure $\mu_{s, t}$ on $B$; we denote by $\left(G_{s, t}, s \leqslant t, t \in T\right)$ a family of independent Gaussian vectors with respective laws $\mu_{s, t}$. Given any finite number of points $\theta=\left\{0=t_{0}<t_{1}<\cdots<t_{n}=T_{0}\right\}$ of $T$, we denote by $\mu^{\theta}$ the law of Gaussian random vectors

$$
Y^{\theta}=\left(\sum_{t_{k} \leqslant t} G_{t_{k}-1, t_{k}}, t_{k} \leqslant t, t \in \theta\right) .
$$


In view of independence, for all $s, t \in \theta$ and $y, z \in B^{\prime}$ we have

$$
\int\langle x(s), y\rangle\langle x(t), z\rangle d \mu^{\theta}(x)=R_{s \wedge t}(y, z) .
$$

This shows that the family $\left(\mu^{\theta}\right)$ where $\theta$ is finite subsets of $T$ is consistent in the sense of Kolmogorov's consistency theorem. So the family $\left(\mu^{\theta}\right)$ defines a Gaussian probability $\mu$ on $B^{T}$ with the required covariance structure. As usual, we can realize $W$ by the identity mapping from $B^{T}$ into $B^{T}$.

In particular, if $R_{t}(y, y)=t R_{1}(y, y)=t \int\langle x, y\rangle^{2} d \mu(x)$, the $B$-valued Wiener process obtained above is called homogeneous and normalized by $\mu$.

This example and its proof is due to Fernique [4]. He has made investigations at length about the problem of existence of continuous versions of some vector-valued Gaussian processes.

As many authors point out that, in a compact metric or pseudometric space $(T, d)$, there are two important quantities measuring the size of $T$ in the $d$ : metric entropy and majorising measure, which are closely related to the continuity and weak convergence for random processes. We denote by $N(T, d, \varepsilon)$ the minimal number of open balls of radius $\varepsilon$ for $d$ that cover $T$. If there exists a probability $m$ in $(T, d)$ such that

$$
\limsup _{\eta \rightarrow 0} \int_{t \in T}^{\eta}\left(\log \frac{1}{m\left(B_{d}(t, \varepsilon)\right)}\right)^{1 / 2} d \varepsilon=0,
$$

where $B_{d}(t, \varepsilon)$ is a ball with center at $t$ and radius $\varepsilon$, then $m$ is called a continuous majorising measure.

Finally, for simplicity and clarity we always assume that $c$ is a positive constant which may vary from line to line and depend on some appropriate parameters.

The present paper is arranged as follows. Section 2 is devoted to the weakly relative compactness of a family of Gaussian measures in $C(T, B)$ and some simple remarks on invariance principle for a sequence of i.i.d. random vectors. In Sections 3 and 4 our subjects will be vector-valued subgaussian processes in the weak sense and vector-valued random processes satisfying the Lipschitz condition, respectively. At the end of this paper, random Fourier series with vector-valued coefficients are taken into account although there is a little gap between our result and one in the scalar setting.

2. Relative compactness of Gaussian measures on $C(T, B)$ and invariance principle. Let $T$ be a compact metric space and $x$ a Gaussian random process on $T$ with continuous sample paths. Fernique [2] proved that the set of separable Gaussian processes $y$ on $T$ such that

$$
\mathbf{E}(y(t)-y(s))^{2} \leqslant \mathbf{E}(x(t)-x(s))^{2}, \quad \forall s, t \in T,
$$

and

$$
\mathbf{E} y^{2}\left(t_{0}\right) \leqslant 1, \quad \text { for some } t_{0} \in T
$$


is relatively compact for the weak convergence of probability measures on the space $C(T)$.

Now we extend the above result to the vector situation. If $X$ is a Gaussian random process with values in a separable Banach space $B$, we denote its covariance by $d_{X}(f, t ; g, s)=\left(\mathbf{E}(\langle f, X(t)\rangle-\langle g, X(s)\rangle)^{2}\right)^{1 / 2}$ for $s, t \in T$ and $f, g \in B^{\prime}$. Gaussian process $X$ is completely specified by $d_{X}$.

Theorem 2.1. Given a continuous Gaussian random process $X$ on $T$ with values in $B$, let $\mathscr{M}$ be the set of the law $\mu_{Y}$ of Gaussian random process $Y$ on $T$ satisfying $d_{Y}(f, t ; g, s) \leqslant d_{X}(f, t ; g, s)$ for any $s, t \in T$ and $f, g \in B^{\prime}$, and for each $f \in B^{\prime}$ there is an element $a$ in $T$ such that $\mathrm{E}\langle f, Y(a)\rangle^{2} \leqslant$ 1. Then $\mathscr{M}$ is relatively compact for the weak convergence of probability measures on $C(T, B)$.

$\mathrm{P}$ r o o f. It is obvious that for each $f \in B^{\prime}$ ( $B^{\prime}$ is of course a separating set of real continuous functions on $B) f \circ \mathscr{M}$ is relatively compact on $C(T)$. Thus by Proposition 1.2 it is enough to show that for any $\varepsilon>0$ there is a compact subset $K \subset B$ such that for each $\mu_{Y} \in \mathscr{M}$,

$$
\mu_{Y}(x: \exists t \in T, x(t) \notin K)<\varepsilon,
$$

i.e., $\mathbf{P}\{\omega: \exists t \in T, Y(\omega, t) \notin K\}<\varepsilon$. For this we need only consider $\mathbf{E} \sup _{t \in T} N_{K}(Y(t))$. The continuity of Gaussian random process $X$ implies that for any positive $\varepsilon$ there exists a convex symmetric and compact set $K$ such that

$$
\mathrm{E} \sup _{t \in T} N_{K}(X(t))<\varepsilon
$$

As we noticed in Section 1, for such a convex symmetric and compact set $K \subset B$ there is a sequence of $f_{n} \in B^{\prime}$ such that $N_{K}(x)=\sup _{n \geqslant 1}\left\langle f_{n}, x\right\rangle$ for all $x \in B$. Since $d_{Y}(f, t ; g, s) \leqslant d_{X}(f, t ; g, s)$, the well-known Slepian's lemma shows

$$
\begin{aligned}
\mathbf{E} \sup _{t \in T} N_{K}(Y(t)) & =\mathbf{E} \sup _{t \in T} \sup _{n \geqslant 1}\left\langle f_{n}, Y(t)\right\rangle \leqslant \mathbf{E} \sup _{t \in T} \sup _{n \geqslant 1}\left\langle f_{n}, X(t)\right\rangle \\
& =\mathbf{E} \sup _{t \in T} N_{K}(X(t)),
\end{aligned}
$$

from which we obtain the required result.

Before concluding this section, we should mention Kuelbs' invariance principle (cf. Kuelbs [8]) for a sequence of i.i.d. random vectors.

Theorem 2.2. Let $\left\{X_{n}, n \geqslant 1\right\}$ be a sequence of independent and symmetric random vectors with the common distribution $\mu$. Suppose

$$
\frac{S_{n}}{\sqrt{n}} \Rightarrow G \text {. }
$$

Construct the partial sum process

$$
X_{n}(t)=\frac{S_{[n t]}}{\sqrt{n}}+\frac{n t-[n t]}{\sqrt{n}} X_{[n t]+1}, \quad 0 \leqslant t \leqslant 1 .
$$


Then $X_{n} \Rightarrow W, W$ is a vector-valued Wiener process generated by $\mu$.

$\mathrm{P}$ r o of. Obviously, the classical Donsker invariance principle can be applied to $\left\langle f, X_{n}(t)\right\rangle$ for each $f \in B^{\prime}$. Thus by Proposition 1.2 it suffices to prove that for any $\varepsilon>0$ there is a compact set $K \subset B$ such that

$$
\sup _{n} \mathrm{P}\left\{\omega: \exists t \in[0,1], X_{n}(t) \notin K\right\}<\varepsilon .
$$

In fact, since $S_{n} / \sqrt{n} \Rightarrow G$, there must be a compact and convex symmetric set $K \subset B$ such that

$$
\sup _{n \geqslant 1} \mathbf{P}\left\{\omega: \frac{S_{n}}{\sqrt{n}} \notin \frac{1}{2} K\right\}<\varepsilon,
$$

from which we deduce by using Levy's inequality

$$
\begin{aligned}
& \sup _{n \geqslant 1} \mathbf{P}\left\{\omega: \sup _{t \in[0,1]} N_{K}\left(X_{n}(t)\right)>1\right\} \leqslant \sup _{n \geqslant 1} \mathbf{P}\left\{\omega: \max _{1 \leqslant k \leqslant n} N_{K}\left(\frac{S_{k}}{\sqrt{n}}\right)>\frac{1}{2}\right\} \\
& \quad+\sup _{n \geqslant 1} \mathbf{P}\left\{\omega: \max _{1 \leqslant k \leqslant n} N_{K}\left(\frac{X_{k}}{\sqrt{n}}\right)>\frac{1}{2}\right\} \\
& \quad \leqslant 4 \sup _{n \geqslant 1} \mathbf{P}\left\{\omega: N_{K}\left(\frac{S_{n}}{\sqrt{n}}\right)>\frac{1}{2}\right\}<4 \varepsilon .
\end{aligned}
$$

Theorem 2.2 now easily follows.

Comparing with Kuelbs' direct method, Lemma 1.2 pays more attention to the topology on any compact set $K \subset B$ : the topology induced on $K$ by the norm topology coincides with the weak topology on $K$, and so has more extensive applications.

3. Vector-valued subgaussian processes. Let us first recall that a real random process $x=(x(t), t \in T)$ is called subgaussian if

$$
\|x(t)-x(s)\|_{\phi_{2}} \leqslant d(s, t)
$$

for any $s, t \in T$, where $\phi_{2}(x)=\exp |x|^{2}-1$ and $\|\cdot\|_{\phi_{2}}$ denotes its Orlicz norm. Heinkel [6] showed that the continuous majorising measure condition is sufficient for $x$ to satisfy the central limit theorem in $C(T)$. (As for the general theory on central limit theorems, see [1], we write CLT for central limit theorem later.)

We can similarly define a vector-valued subgaussian process $X=(X(t)$, $t \in T)$ in the strong sense, that is,

$$
\|X(t)-X(s)\|_{\phi_{2}} \leqslant d(s, t), \quad \forall s, t \in T .
$$

Although the continuous majorising measure condition can still guarantee the existence of modification with continuous sample paths, the CLT does not hold for $X$ any longer. 
For example, let $\left(x_{i}, i \geqslant 1\right)$ be a sequence of elements in $C(T, B)$ and $\left(\varepsilon_{i}, i \geqslant 1\right)$ a Rademacher sequence, define $X(t)=\sum_{i=1}^{\infty} \varepsilon_{i} x_{i}(t)$, for $t \in T$. By [9] we have

$$
\|X(s)-X(t)\|_{\phi_{2}} \leqslant 8\left(\mathrm{E}\left\|\sum_{i=1}^{\infty} \varepsilon_{i}\left(x_{i}(s)-x_{i}(t)\right)\right\|^{2}\right)^{1 / 2}=: d(s, t) .
$$

Only when $Y(t)=\sum_{i=1}^{\infty} g_{i} x_{i}(t), t \in T$, is also a.s. continuous can $X$ as a random vector in $C(T, B)$ satisfy the CLT.

Now a vector-valued random process $X=(X(t), t \in T)$ with values in $B$ is said to be subgaussian in the weak sense if for each $f \in B^{\prime}$ there is a continuous distance $d_{f}(s, t)$ with respect to $d$ on $T$ such that

$$
\|\langle f, X(s)\rangle-\langle f, X(t)\rangle\|_{\phi_{2}} \leqslant d_{f}(s, t), \quad \forall s, t \in T .
$$

Obviously, $\|\langle f, X(s)\rangle-\langle f, X(t)\rangle\|_{\phi_{2}} \leqslant\|f\|\|X(s)-X(t)\|_{\phi_{2}}$.

On the other hand, $X=(X(t), t \in T)$ is called uniformly $\gamma$-subgaussian if there is a Gaussian vector $G$ in $B$ such that

$$
\mathbf{E} \exp \langle f, X(t)\rangle \leqslant \mathbf{E} \exp \langle f, G\rangle, \quad \forall t \in T, f \in B^{\prime} .
$$

See [6] for details on $\gamma$-subgaussian and subgaussian vectors.

Our main result on vector-valued subgaussian process is as follows.

Theorem 3.1. Suppose that $X=(X(t), t \in T)$ is both a subgaussian in the weak sense and a uniformly $\gamma$-subgaussian process. If there exists a probability measure $m$ on $(T, d)$ such that

$$
\lim _{\eta \rightarrow 0} \sup _{f \in B^{\prime}} \sup _{t \in T} \int_{0}^{\eta}\left(\log \frac{1}{m\left(B_{d_{f}}(t, \varepsilon)\right)}\right)^{1 / 2} d \varepsilon=0
$$

and $\sup _{t \in T} \int_{0}^{\infty}\left(\log \left(m\left(B_{d_{f}}(t, \varepsilon)\right)\right)^{-1}\right)^{1 / 2} d \varepsilon$ is continuous with respect to the topology of the compact convergence on $B$, then $X$ is a.s. continuous on $T$ and satisfies the $C L T$ in $C(T, B)$.

P r o of. We first note that for any $s, t \in T$ and $f, g \in B^{\prime}$,

$$
\begin{aligned}
d_{X}(f, t ; g, s)= & \|\langle f, X(t)\rangle-\langle g, X(s)\rangle\|_{\phi_{2}} \leqslant\|\langle f, X(s)\rangle-\langle g, X(s)\rangle\|_{\phi_{2}} \\
& +\|\langle f, X(s)\rangle-\langle f, X(t)\rangle\|_{\phi_{2}} \\
\leqslant & 5\left(\mathbf{E}(f-g, G\rangle^{2}\right)^{1 / 2}+d_{f}(s, t)=: d_{G}(f, g)+d_{f}(s, t) .
\end{aligned}
$$

So, for any $\varepsilon>0, B_{d_{G}}(f, \varepsilon / 2) \times B_{d_{f}}(t, \varepsilon / 2) \subset B_{d_{X}}((f, t) ; \varepsilon)$. Since $G$ is a Gaussian vector with values in $B$ and $B_{1}^{\prime}$ is $\sigma\left(B, B^{\prime}\right)$ compact and metrizable, 
by Talagrand's theorem [9] there is a probability measure $\mu$ on $B_{1}^{\prime}$ such that

$$
\sup _{f \in B_{1}^{\prime}} \int_{0}^{\infty}\left(\log \frac{1}{\mu\left(B_{d_{G}}(f, \varepsilon)\right)}\right)^{1 / 2} d \varepsilon \leqslant c E\|G\| .
$$

Let $\pi=\mu \times m$, then $m\left(B_{d_{f}}(t, \varepsilon / 2)\right) \mu\left(B_{d_{G}}(f, \varepsilon / 2)\right) \leqslant \pi\left(B_{d_{X}}((f, t) ; \varepsilon)\right)$, and hence

$$
\begin{aligned}
& \sup _{f \in B_{1}^{\prime}} \sup _{t \in T} \int_{0}^{\infty}\left(\log \frac{1}{\pi\left(B_{d_{X}}((f, t), \varepsilon)\right)}\right)^{1 / 2} d \varepsilon \\
& \leqslant \sup _{f \in B_{1}^{\prime}} \sup _{t \in T} \int_{0}^{\infty}\left(\log \frac{1}{m\left(B_{d_{f}}\left(t, \frac{\varepsilon}{2}\right)\right)}\right)^{1 / 2} d \varepsilon \\
& \quad+\sup _{f \in B_{1}^{\prime}} \int_{0}^{\infty}\left(\log \frac{1}{\mu\left(B_{d_{G}}\left(f, \frac{\varepsilon}{2}\right)\right)}\right)^{1 / 2} d \varepsilon \\
& \leqslant c\left(\mathrm{E}\|G\|+\sup _{f \in B_{1}^{\prime}} \sup _{t \in T} \int_{0}^{\infty}\left(\log \frac{1}{m\left(B_{d_{f}}(t, \varepsilon)\right)}\right)^{1 / 2} d \varepsilon\right) .
\end{aligned}
$$

Now we can use Fernique's theorem [2] to obtain

$$
\begin{aligned}
\mathbf{E} \sup _{t \in T}\|X(t)\| & =\mathbf{E} \sup _{t \in T} \sup _{f \in B_{1}^{\prime}}\langle f, X(t)\rangle \\
& \leqslant c\left(\mathbf{E}\|G\|+\sup _{f \in B_{1}^{\prime}} \sup _{t \in T} \int_{0}^{\infty}\left(\log \frac{1}{m\left(B_{d_{f}}(t, \varepsilon)\right)}\right)^{1 / 2} d \varepsilon\right) .
\end{aligned}
$$

On the other hand, for any $\varepsilon>0$ there is a convex symmetric and compact set $K \subset B$ such that

$$
\mathbf{E} N_{K}(G)+\sup _{f \in K^{0}} \sup _{t \in T} \int_{0}^{\infty}\left(\log \frac{1}{m\left(B_{d_{f}}(t, \varepsilon)\right)}\right)^{1 / 2} d \varepsilon<\varepsilon .
$$

For such a compact set $K$ there exists an increasing sequence $N_{k}$ of continuous seminorms, converging simply to $N_{K}$ in $B$, and $\left(B, N_{k}\right)$ is a separable Banach space.

By repeating the previous proof for $\left(B, N_{k}\right)$ and taking the limit, we also have

$$
\mathbf{E} \sup _{t \in T} N_{K}(X(t))<c \varepsilon .
$$

Thus, according to Theorem 4.2.1 in [4], $X$ has a modification with continuous sample paths since each real-valued random process $f(X)$ is a.s. continuous on $T$ for $f \in B_{1}^{\prime}$.

To prove that $X$ satisfies the CLT, without loss of generality we can assume that $X$ is symmetric and $\left\{X, X_{n}, n \geqslant 1\right\}$ are independent identically distributed. Consider the normed sums $Z_{n}=n^{-1 / 2} \sum_{i=1}^{n} X_{i}$. 
It is not hard to verify that

$$
\mathbf{E} \exp \left\langle f, Z_{n}(t)\right\rangle \leqslant \mathbf{E} \exp \langle f, G\rangle, \quad f \in B_{1}^{\prime}, t \in T,
$$

and

$$
\left\|\left\langle f, Z_{n}(t)\right\rangle-\left\langle f, Z_{n}(s)\right\rangle\right\|_{\phi_{2}} \leqslant c d_{f}(s, t) .
$$

Thus one can similarly prove that for any $\varepsilon>0$ there is a convex symmetric and compact $K \subset B$ such that

$$
\sup _{n \geqslant 1} \mathbf{E} \sup _{t \in T} N_{K}\left(Z_{n}(t)\right)<\varepsilon
$$

For each $f \in B^{\prime}$, Heinkel [7] tells us that $f(X)$ satisfies the CLT in $C(T)$. Hence we deduce that $\left\{Z_{n}, n \geqslant 1\right\}$ is relatively compact by using Proposition 1.2 again.

As for the uniqueness of the limit, it is enough to notice Singer Theorem and the CLT in finite-dimensional spaces. The proof of Theorem 3.1 is completed.

4. Vector-valued random Lipschitz processes. We will begin our arguments with the following two lemmas. The first lemma is Lemma 7.13 in [1] and the second is just the formula (4.12) in [9].

Lemma 4.1. Suppose that $(T, d)$ is a compact metric space and

$$
\int_{0}^{\infty}(\log N(T, d, \varepsilon))^{1 / 2} d \varepsilon<\infty
$$

Then there is another continuous pseudometric $d^{\prime}$ such that $d \leqslant d^{\prime}, d / d^{\prime} \rightarrow 0$ and $\int_{0}^{\infty}\left(\log N\left(T, d^{\prime}, \varepsilon\right)\right)^{1 / 2} d \varepsilon<\infty$.

Lemma 4.2. Suppose that $X$ is a subgaussian vector in $B$. Then for any $t>0$,

$$
\mathbf{P}\{\|X\|>t\} \leqslant 2 \exp \left(-\frac{t^{2}}{32 \mathrm{E}\|X\|^{2}}\right) .
$$

Our object in this section is to establish the following

Theorem 4.1. Suppose that $B$ is the space of type $2, X=(X(t), t \in T)$ is a mean zero $B$-valued random process satisfying Lipschitz condition, i.e.,

$$
\|X(t)-X(s)\| \leqslant M d(s, t),
$$

where $M$ is a real-valued random variable with finite variance.

If $\int_{0}^{\infty}(\log N(T, d, \varepsilon))^{1 / 2} d \varepsilon<\infty$, then $X$ satisfies the $C L T$ on $C(T, B)$.

$\mathrm{P}$ r o of. Our hypotheses guarantee that $f(X)$ satisfies the CLT for each $f \in B^{\prime}$ and multi-dimensional CLT remains true. This is a consequence of Theorem 14.2 in [9]. 
The key to the proof of Theorem 4.1 is to prove that for each $\varepsilon>0$ and $\eta>0$ there are a positive constant $a$ and a finite-dimensional closed subspace $F$ such that

$$
\sup _{n \geqslant 1} \mathbf{P}\left\{\omega: \sup _{t \in T}\left\|\frac{1}{\sqrt{n}} \sum_{i=1}^{n} \varepsilon_{i} X_{i}(t)\right\|>a\right\}<\varepsilon
$$

and

$$
\sup _{n \geqslant 1} \mathbf{P}\left\{\omega: \sup _{t \in T} q_{F}\left(\frac{1}{\sqrt{n}} \sum_{i=1}^{n} \varepsilon_{i} X_{i}(t)\right)>\eta\right\}<\varepsilon .
$$

Here $\left\{X, X_{n}, n \geqslant 1\right\}$ is a sequence of independent identically distributed random processes and $\left\{\varepsilon_{n}, n \geqslant 1\right\}$ is a Rademacher sequence independent of $\left\{X_{n}, n \geqslant 1\right\}$. The two statements will be proved in analogous ways: using truncation.

In fact, since $\mathbf{E} M^{2}<\infty$, for any $\varepsilon>0$ there is a positive constant $a_{0}$ such that

$$
\sup _{n \geqslant 1} P\left\{\omega: \frac{1}{n} \sum_{i=1}^{n} \sup _{s \neq t} \frac{\left\|X_{i}(s)-X_{i}(t)\right\|^{2}}{a_{0}^{2} d^{2}(s, t)} \geqslant 1\right\} \leqslant \frac{E M^{2}}{a_{0}^{2}}<\frac{\varepsilon}{2} .
$$

For such a fixed $a_{0}$ it easily follows from Lemma 4.2 that

$$
\begin{aligned}
& \mathbf{E}_{X} \mathbf{P}_{\varepsilon}\{\| \frac{1}{\sqrt{n}} \sum_{i=1}^{n} \varepsilon_{i}\left(X_{i}(t)-X_{i}(s)\right) \\
&\left.\times I\left(\frac{1}{n} \sum_{i=1}^{n} \sup _{s \neq t} \frac{\left\|X_{i}(s)-X_{i}(t)\right\|^{2}}{a_{0}^{2} d^{2}(s, t)} \leqslant 1\right) \|>u a_{0} d(s, t)\right\} \\
& \leqslant 2 \mathbf{E}_{X} \exp \left(-u^{2}\left[32 \mathbf{E}_{\varepsilon} \| \frac{1}{\sqrt{n}} \sum_{i=1}^{n} \varepsilon_{i}\left(X_{i}(t)-X_{i}(s)\right)\right.\right. \\
&\left.\left.\quad \times I\left(\frac{1}{n} \sum_{i=1}^{n} \sup _{s \neq t} \frac{\left\|X_{i}(s)-X_{i}(t)\right\|^{2}}{a_{0}^{2} d^{2}(s, t)} \leqslant 1\right) \|^{2}\right]^{-1}\right) \\
& \leqslant 2 \exp \left(-\frac{u^{2}}{32 c}\right),
\end{aligned}
$$

where $c$ is an absolute constant in the definition of the space of type 2. In other words,

$$
\begin{aligned}
& \left\|\frac{1}{\sqrt{n}} \sum_{i=1}^{n} \varepsilon_{i}\left(X_{i}(t)-X_{i}(s)\right) I\left(\frac{1}{n} \sum_{i=1}^{n} \sup _{s \neq t} \frac{\left\|X_{i}(s)-X_{i}(t)\right\|^{2}}{a_{0}^{2} d^{2}(s, t)} \leqslant 1\right)\right\|_{\phi_{2}} \\
& \quad \leqslant c a_{0} d(s, t) .
\end{aligned}
$$


Thus we have

$$
\begin{aligned}
& \mathbf{E} \sup _{t \in T}\left\|\frac{1}{\sqrt{n}} \sum_{i=1}^{n} \varepsilon_{i} X_{i}(t) I\left(\frac{1}{n} \sum_{i=1}^{n} \sup _{s \neq t} \frac{\left\|X_{i}(s)-X_{i}(t)\right\|^{2}}{a_{0}^{2} d^{2}(s, t)} \leqslant 1\right)\right\| \\
& \leqslant c a_{0} \int_{0}^{\infty}(\log N(T, d, \varepsilon))^{1 / 2} d \varepsilon,
\end{aligned}
$$

from which there exists a positive constant $a$ such that

$$
\begin{aligned}
\sup _{n \geqslant 1} \mathbf{P}\left\{\omega: \sup _{t \in T} \|\right. & \frac{1}{\sqrt{n}} \sum_{i=1}^{n} \varepsilon_{i} X_{i}(t) \\
& \left.\times I\left(\frac{1}{n} \sum_{i=1}^{n} \sup _{s \neq t} \frac{\left\|X_{i}(s)-X_{i}(t)\right\|^{2}}{a_{0}^{2} d^{2}(s, t)} \leqslant 1\right) \| \geqslant a\right\} \leqslant \frac{\varepsilon}{2} .
\end{aligned}
$$

Now, the first statement holds.

The proof of the second one is similar but to use Lemma 4.1. Let $d^{\prime}$ be as in Lemma 4.1, and define

$$
Y(s, t)= \begin{cases}\frac{X(t)-X(s)}{d^{\prime}(s, t)} & \text { for } s \neq t \\ 0 & \text { otherwise. }\end{cases}
$$

It is easy to see that $Y(s, t)$ is a.s. in $C(T \times T, B)$ since $d / d^{\prime} \rightarrow 0$.

So, for each $\varepsilon>0$ and $\eta>0$ there must be a finite-dimensional closed subspace $F$ such that

$$
\mathbf{P}\left\{\omega: \exists s \neq t, q_{F}(Y(s, t))>\eta\right\}<\varepsilon .
$$

Further, by the dominated convergence theorem it is not hard to conclude that for any $\varepsilon>0$ and $\eta>0$ there must be a finite-dimensional closed subspace $F$ such that

$$
\sup _{n \geqslant 1} \mathbf{P}\left\{\omega: \frac{1}{n} \sum_{i=1}^{n} \sup _{s \neq t} \frac{q_{F}\left(X_{i}(s)-X_{i}(t)\right)^{2}}{\varepsilon^{2} \eta^{2}\left(d^{\prime}(s, t)\right)^{2}} \geqslant 1\right\}<\frac{\varepsilon}{2}
$$

Analogously,

$$
\begin{aligned}
\mathbf{E}_{X} \mathbf{P}_{\varepsilon} & \left\{q _ { F } \left(\frac{1}{\sqrt{n}} \sum_{i=1}^{n} \varepsilon_{i}\left(X_{i}(t)-X_{i}(s)\right)\right.\right. \\
& \left.\times I\left(\frac{1}{n} \sum_{i=1}^{n} \sup _{s \neq t} \frac{q_{F}\left(X_{i}(s)-X_{i}(t)\right)^{2}}{\varepsilon^{2} \eta^{2}\left(d^{\prime}(s, t)\right)^{2}} \leqslant 1\right)>u a_{0} d(s, t)\right\} \\
\leqslant & 2 \mathbf{E}_{X} \exp \left(-u^{2}\left(3 2 \mathrm { E } _ { \varepsilon } q _ { F } \left(\frac{1}{\sqrt{n}} \sum_{i=1}^{n} \varepsilon_{i}\left(X_{i}(t)-X_{i}(s)\right)\right.\right.\right.
\end{aligned}
$$




$$
\begin{aligned}
& \left.\left.\left.\times I\left(\frac{1}{n} \sum_{i=1}^{n} \sup _{s \neq t} \frac{q_{F}\left(X_{i}(s)-X_{i}(t)\right)^{2}}{\varepsilon^{2} \eta^{2}\left(d^{\prime}(s, t)\right)^{2}} \leqslant 1\right)\right)^{2}\right)^{-1}\right) \\
& \leqslant 2 \exp \left(-\frac{u^{2}}{32 c}\right) .
\end{aligned}
$$

Here in the last step we use the fact that any quotient space of type 2 remains to be of type 2 and has the same constant.

So, we have

$$
\begin{aligned}
& \mathbf{E} \sup _{t \in T} q_{F}\left(\frac{1}{\sqrt{n}} \sum_{i=1}^{n} \varepsilon_{i} X_{i}(t) I\left(\frac{1}{n} \sum_{i=1}^{n} \sup _{s \neq t} \frac{q_{F}\left(X_{i}(s)-X_{i}(t)\right)^{2}}{\varepsilon^{2} \eta^{2}\left(d^{\prime}(s, t)\right)^{2}} \leqslant 1\right)\right) \\
& \leqslant c \int_{0}^{\infty}\left(\log N\left(T, \varepsilon \eta d^{\prime}, u\right)\right)^{1 / 2} d u \\
& \leqslant c \varepsilon \eta \int_{0}^{\infty}\left(\log N\left(T, d^{\prime}, u\right)\right)^{1 / 2} d u .
\end{aligned}
$$

Thus the required result (4.4) can be proved by noting Lemma 4.1. The proof of Theorem 4.3 is concluded.

5. Random Fourier series with vector-valued coefficients. Random Fourier series with real coefficients have been studied in details by Marcus and Pisier [10] and so on. It appears many elegant results and applications to harmonic analysis. Up to now, however, the results concerning random Fourier series with vector-valued coefficients are still fragmentary. Let us first recall some backgrounds.

Let $G$ be a compact Abel group, $\Gamma=\left\{\gamma_{n}, n \geqslant 1\right\}$ a countable subset of its character group. Suppose that $\left\{g_{n}, n \geqslant 1\right\}$ is a sequence of independent standard Gaussian random variables, $\left\{\varepsilon_{n}, n \geqslant 1\right\}$ is a Rademacher sequence, $\left\{\xi_{n}, n \geqslant 1\right\}$ is a sequence of independent symmetric real random variables with $\mathbf{E} \xi_{n}^{2}=1$ and $\inf _{n \geqslant 1} \mathbf{E}\left|\xi_{n}\right|>0,\left\{a_{n}, n \geqslant 1\right\}$ are real numbers satisfying $\sum_{n=1}^{\infty}\left|a_{n}\right|^{2}<\infty$. Define three Fourier series on $G$ as follows:

$$
\begin{aligned}
& x(t)=\sum_{n=1}^{\infty} a_{n} \xi_{n} \gamma_{n}(t), \quad y(t)=\sum_{n=1}^{\infty} a_{n} g_{n} \gamma_{n}(t) \\
& z(t)=\sum_{n=1}^{\infty} a_{n} \varepsilon_{n} \gamma_{n}(t)
\end{aligned}
$$

and define $d(s, t)=\left(\sum_{n=1}^{\infty}\left|a_{n}\right|^{2}\left|\gamma_{n}(s)-\gamma_{n}(t)\right|^{2}\right)^{1 / 2}$ for $s, t \in G$. Main results in $[10]$ show that

$$
\mathbf{E}\left\|\sum_{n=1}^{\infty} a_{n} \xi_{n} \gamma_{n}\right\| \approx \mathbf{E}\left\|\sum_{n=1}^{\infty} a_{n} g_{n} \gamma_{n}\right\| \approx \mathbf{E}\left\|\sum_{n=1}^{\infty} a_{n} \varepsilon_{n} \gamma_{n}\right\|
$$


(all of them are equivalent to $\int_{0}^{\infty}(\log N(G, d, \varepsilon))^{1 / 2}<\infty$ ) although $C(G)$, the set of all bounded continuous complex functions on $G$, is of no finite cotype. As an important corollary, $x$ satisfies the CLT on $C(G)$ and the limit is just $y$.

Similar problems naturally arise when real coefficients $\left\{a_{n}, n \geqslant 1\right\}$ are replaced by a sequence of vectors $\left\{x_{n}, n \geqslant 1\right\}$ in a separable Banach space $B$. If $\sum_{n=1}^{\infty} x_{n} \xi_{n}, \sum_{n=1}^{\infty} x_{n} g_{n}$, and $\sum_{n=1}^{\infty} x_{n} \varepsilon_{n}$ a.s. converge respectively, we can define respectively by the comparison convergence theorem for random series

$$
\begin{aligned}
X(t) & =\sum_{n=1}^{\infty} x_{n} \xi_{n} \gamma_{n}(t), \quad Y(t)=\sum_{n=1}^{\infty} x_{n} g_{n} \gamma_{n}(t), \\
Z(t) & =\sum_{n=1}^{\infty} x_{n} \varepsilon_{n} \gamma_{n}(t) .
\end{aligned}
$$

Fernique [3] first showed that a vector-valued stationary Gaussian process $Y$ has a modification with continuous sample paths if and only if the mapping $y \rightarrow \mathbf{E} \sup _{t \in G}\langle y, Y(t)\rangle$ from $B^{\prime}$ to $C$ is continuous with respect to the topology of the compact convergence on $B$. On the other hand, we have the following relation (cf. [9, Corollary 13.14])

$$
\begin{aligned}
& c\left(\mathbf{E}\left\|\sum_{n=1}^{\infty} \varepsilon_{n} x_{n}\right\|+\sup _{f \in B_{1}^{\prime}} \mathbf{E} \sup _{t \in G} \sum_{n=1}^{\infty} \varepsilon_{n}\left\langle f, x_{n}\right\rangle \gamma_{n}(t)\right) \\
& \quad \leqslant \mathbf{E} \sup _{t \in G}\left\|\sum_{n=1}^{\infty} \varepsilon_{n} x_{n} \gamma_{n}(t)\right\| \leqslant \mathbf{E} \sup _{t \in G}\left\|\sum_{n=1}^{\infty} g_{n} x_{n} \gamma_{n}(t)\right\| \\
& \quad \leqslant c\left(\mathbf{E}\left\|\sum_{n=1}^{\infty} g_{n} x_{n}\right\|+\sup _{f \in B_{1}^{\prime}} \mathbf{E} \sup _{t \in G} \sum_{n=1}^{\infty} g_{n}\left\langle f, x_{n}\right\rangle \gamma_{n}(t)\right) .
\end{aligned}
$$

Thus it is easily seen that if $\sum_{n=1}^{\infty} g_{n} x_{n}<\infty$ a.s., then $\sum_{n=1}^{\infty} \varepsilon_{n} x_{n} \gamma_{n}(t)$ and $\sum_{n=1}^{\infty} g_{n} x_{n} \gamma_{n}(t)$ converge uniformly a.s. simultaneously, i.e., they have the same continuity. Of course, when $Y=\sum_{n=1}^{\infty} x_{n} g_{n} \gamma_{n}(t)$ converges uniformly a.s., $Z$ also satisfies the CLT in $C(G, B)$.

The problems remain open, however, for a sequence of random variables $\left\{\xi_{n}, n \geqslant 1\right\}$, both Gaussian and Rademacher. Next we give our results at this area.

Theorem 5.1. Suppose that $B$ is the space of cotype $q(2 \leqslant q<\infty)$, $1 / p+1 / q=1 .\left\{\xi_{n}, n \geqslant 1\right\}$ is a sequence of independent symmetric real random variables with $\mathbf{E} \xi_{n}^{2}=1$ and $\inf _{n} \mathbf{E}|\xi|>0, \sup _{n} \mathbf{E}\left|\xi_{n}\right|^{2 q}<\infty$. Further, assume that $\mathbf{E}\left\|\sum_{n=1}^{\infty} \xi_{n} x_{n}\right\|<\infty$. Define

$$
X(t)=\sum_{n=1}^{\infty} \xi_{n} x_{n} \gamma_{n}(t)
$$


and

$$
d(s, t)=\sup _{f \in B_{1}^{\prime}}\left(\sum_{n=1}^{\infty}\left|\left\langle f, x_{n}\right\rangle\right|^{p}\left|\gamma_{n}(s)-\gamma_{n}(t)\right|^{2 p}\right)^{1 /(2 p)}
$$

If

$$
\int_{0}^{\infty}(\log N(G, d, \varepsilon))^{1 / 2} d \varepsilon<\infty
$$

then $X(t)$ is a.s. continuous on $G$.

In addition, if $\sum_{n=1}^{\infty} \xi_{n} x_{n}$ satisfies the $C L T$ in $B$, then $X$ satisfies the $C L T$ in $C(G, B)$.

P r o of. First note that $\sum_{n=1}^{\infty}\left\|x_{n}\right\|^{q}<\infty$ due to the hypotheses, and for each $f \in B_{1}^{\prime}$

$$
\begin{aligned}
& \left(\sum_{n=1}^{\infty}\left|\left\langle f, x_{n}\right\rangle\right|^{2}\left|\gamma_{n}(s)-\gamma_{n}(t)\right|^{2}\right)^{1 / 2} \\
& \quad \leqslant\left(\sum_{n=1}^{\infty}\left\|x_{n}\right\|^{q}\right)^{1 /(2 q)}\left(\sum_{n=1}^{\infty}\left|\left\langle f, x_{n}\right\rangle\right|^{p}\left|\gamma_{n}(s)-\gamma_{n}(t)\right|^{2 p}\right)^{1 /(2 p)} \\
& \quad=\left(\sum_{n=1}^{\infty}\left\|x_{n}\right\|^{q}\right)^{1 /(2 q)} d(s, t)
\end{aligned}
$$

by Hölder inequality.

Since $\int_{0}^{\infty}(\log N(G, d, \varepsilon))^{1 / 2} d \varepsilon<\infty$, then $f(X)$ is a.s. continuous on $G$ and satisfies the CLT in $C(G)$ for each $f \in B_{1}^{\prime}$.

Next define

$$
d_{f}(s, t)=\left(\sum_{n=1}^{\infty} \xi_{n}^{2}\left|\left\langle f, x_{n}\right\rangle\right|^{2}\left|\gamma_{n}(s)-\gamma_{n}(t)\right|^{2}\right)^{1 / 2} .
$$

Then using (5.4) and noting $B$ is of cotype $q$ space, we have

$$
\begin{aligned}
& \mathbf{E} \sup _{t \in G}\left\|\sum_{n=1}^{\infty} \xi_{n} x_{n} \gamma_{n}(t)\right\| \\
& \leqslant c\left(\mathbf{E}\left\|\sum_{n=1}^{\infty} \xi_{n} x_{n}\right\|+\mathbf{E}_{\xi} \sup _{f \in B_{1}^{\prime}} \mathbf{E}_{\varepsilon} \sup _{t \in G} \sum_{n=1}^{\infty} \xi_{n} \varepsilon_{n}\left\langle f, x_{n}\right\rangle \gamma_{n}(t)\right) \\
& \leqslant c\left(\mathbf{E}\left\|\sum_{n=1}^{\infty} \xi_{n} x_{n}\right\|+\mathrm{E}_{\xi} \sup _{f \in B_{1}^{\prime}} \int_{0}^{\infty}\left(\log N\left(G, d_{f}, \varepsilon\right)\right)^{1 / 2} d \varepsilon\right) \\
& \leqslant c\left(\mathbf{E}\left\|\sum_{n=1}^{\infty} \xi_{n} x_{n}\right\|+\mathbf{E}_{\xi} \int_{0}^{\infty}\left(\log N\left(G, \sup _{f \in B_{1}^{\prime}} d_{f}(s, t), \varepsilon\right)\right)^{1 / 2} d \varepsilon\right)
\end{aligned}
$$


Clearly, $\sup _{f \in B_{1}^{\prime}} d_{f}(s, t)$ is a translation invariant metric, from Lemma 3.11 in [11] it follows that

$$
\begin{aligned}
& \mathbf{E}_{\xi} \int_{0}^{\infty}\left(\log N\left(G, \sup _{f \in B_{1}^{\prime}} d_{f}(s, t), \varepsilon\right)\right)^{1 / 2} d \varepsilon \\
& \leqslant c\left(\mathbf{E}_{\xi} \sup _{f \in B_{1}^{\prime}}\left(\sum_{n=1}^{\infty} \xi_{n}^{2}\left|\left\langle f, x_{n}\right)\right|^{2}\right)^{1 / 2}\right. \\
& \left.\quad+\int_{0}^{\infty}\left(\log N\left(G, \mathbf{E}_{\xi} \sup _{f \in B_{1}^{\prime}} d_{f}(s, t), \varepsilon\right)\right)^{1 / 2} d \varepsilon\right) \\
& \leqslant c\left(\mathbf{E}_{\xi}\left(\sum_{n=1}^{\infty}\left\|x_{n}\right\|^{q}\left|\xi_{n}\right|^{2 q}\right)^{1 /(2 q)} \sup _{f \in B_{1}^{\prime}}\left(\sum_{n=1}^{\infty}\left|\left\langle f, x_{n}\right\rangle\right|^{p}\right)^{1 /(2 p)}\right. \\
& \left.\quad+\int_{0}^{\infty}\left(\log N\left(G, \mathbf{E}_{\xi}\left(\sum_{n=1}^{\infty}\left\|x_{n}\right\|^{q}\left|\xi_{n}\right|^{2 q}\right)^{1 /(2 q)} d, \varepsilon\right)\right)^{1 / 2} d \varepsilon\right) \\
& \leqslant c\left(\left(\sum_{n=1}^{\infty}\left\|x_{n}\right\|^{q}\right)^{1 /(2 q)} \sup _{f \in B_{1}^{\prime}}\left(\sum_{n=1}^{\infty}\left|\left\langle f, x_{n}\right\rangle\right|^{p}\right)^{1 /(2 p)}\right. \\
& \left.\quad+\int_{0}^{\infty}(\log N(G, d, \varepsilon))^{1 / 2} d \varepsilon\right) .
\end{aligned}
$$

Similarly,

$$
\begin{gathered}
\underset{t \in G}{\mathbf{E} \sup _{t \in G}}\left\|\sum_{n>N}^{\infty} \xi_{n} x_{n} \gamma_{n}(t)\right\| \\
\leqslant c\left(\mathrm{E}\left\|\sum_{n>N}^{\infty} \xi_{n} x_{n}\right\|+\left(\sum_{n>N}^{\infty}\left\|x_{n}\right\|^{q}\right)^{1 /(2 q)}\left(\sup _{f \in B_{1}^{\prime}}\left(\sum_{n>N}^{\infty}\left|\left\langle f, x_{n}\right\rangle\right|^{p}\right)^{1 /(2 p)}\right.\right. \\
\left.\quad+\int_{0}^{\infty}\left(\log N\left(G, d_{N}, \varepsilon\right)\right)^{1 / 2} d \varepsilon\right)
\end{gathered}
$$

where $d_{N}(s, t)=\sup _{f \in B_{1}^{\prime}}\left(\sum_{n>N}^{\infty}\left|\left\langle f, x_{n}\right\rangle\right|^{p}\left|\gamma_{n}(s)-\gamma_{n}(t)\right|^{2 p}\right)^{1 /(2 p)}$.

Thus $X(t)$ is a.s. continuous on $G$.

If $\sum_{n=1}^{\infty} \xi_{n} x_{n}$ satisfies the CLT in $B$, in order to prove that $X$ satisfies the CLT in $C(G, B)$ it suffices to consider $Z_{m}=m^{-1 / 2} \sum_{i=1}^{m} \sum_{n=1}^{\infty} \xi_{n}^{i} x_{n} \gamma_{n}$, where $\left\{\xi_{n}^{i}, n \geqslant 1\right\}, i=1,2, \ldots$, is a sequence of independent sequences with the same distributions as $\left\{\xi_{n}, n \geqslant 1\right\}$. By some simple calculations,

$$
\mathbf{E}\left|\frac{1}{\sqrt{m}} \sum_{i=1}^{m} \xi_{n}^{i}\right|^{2}=1, \quad \sup _{m} \sup _{n} \mathbf{E}\left|\frac{1}{\sqrt{m}} \sum_{i=1}^{m} \xi_{n}^{i}\right|^{2 q}<\infty .
$$


Therefore we obtain as above

$$
\sup _{m \geqslant 1} \mathbf{E} \sup _{t \in G}\left\|Z_{m}\right\|<\infty
$$

and

$$
\lim _{N \rightarrow \infty} \sup _{m \geqslant 1} \mathbf{E} \sup _{t \in G}\left\|\frac{1}{\sqrt{m}} \sum_{i=1}^{m} \sum_{n>N}^{\infty} \xi_{n}^{i} x_{n} \gamma_{n}(t)\right\|=0 .
$$

We now can complete the proof of Theorem 5.1 by using Lemma $1.2(1.2)$ and (1.3).

Acknowledgement. This is part of the author's Ph. D. dissertation at Fudan University. The author would like to express his gratitude to Professors Z. Y. Lin and C. R. Lu for their encouragement and help. The author also wishes to thank Professor X. Fernique for providing the author with his manuscripts.

\section{REFERENCES}

1. Araujo A., Gine E. The Central Limit Theorem for Real and Banach Space Valued Random Variables. New York: Wiley, 1980.

2. Fernique $X$. Regularité des trajectoires des fonctions aléatoires gaussiennes. - Lect. Notes in Math., 1975, v. 490, p. 1-96.

3. Fernigue $X$. Sur la convergence étroite des mesures gaussiennes. - Z Wahrscheinlichkeitstheor. verw. Geb., 1985, B. 68, S. 331-336.

4. Fernique $X$. Fonctions aléatoires à valeurs dans les espaces lusiniens. - Exposition. Math., 1990, N: 8, p. 289-364.

5. Fernique $X$. Convergence en loi de fonctions aléatoires continues or càdlàg, propriétés de compacité des lois. - Lect. Notes in Math., 1991, v. 1485, p. 178-195.

6. Fukuda F. Exponential inequality of sub-gaussian vectors. - Probab. Theory Relat. Fields, 1990, v. 85, p. 505-521.

7. Heinkel $B$. Mesures majorantes et théorème de la limite centrale dans $C(S)$. Z. Wahrscheinlichkeitstheor. verw. Geb., 1977, B. 38, S. 339-351.

8. Kuelbs $J$. The invariance principle for Banach space valued random variable. J. Multivariate Anal., 1973, v. 3, p. 161-172.

9. Ledoux M., Talagrand M. Probability in Banach Spaces. Berlin: Springer-Verlag, 1991.

10. Marcus M. B., Pisier G. Random Fourier Series with Applications to Harmonic Analysis. Princeton: Princeton Univ. Press, Univ. of Tokyo Press, 1981.

11. Marcus $M$. B., Pisier G. Characterizations of almost surely continuous p-stable random Fourier series and strongly stationary processes. - Acta Math., 1984, v. 152, p. 245-301.

12. Singer I. Sur les applications linéaires intégrales des espaces de fonctions continues I. - Rev. Math. Pur. Appl., 1959, v. 4, p. 391-401. 